\title{
Ảnh hưởng của giá trị tiêu dùng đến ý định chấp nhận sản phẩm mới: Nghiên cứu nhóm sản phẩm điện tử cá nhân tại thị trường Thành phố Hồ Chí Minh
}

\section{The effects of consumption values on new product adoption intention: A study of personal electronic industry in Ho Chi Minh City}

\author{
Dư Thị Chung ${ }^{1 *}$, Ngô Thị Thu ${ }^{1}$, Trần Văn Thi ${ }^{1}$ \\ ${ }^{1}$ Trường Đại học Tài chính-Marketing, Việt Nam \\ *Tác giả liên hệ, Email: duchung@ufm.edu.vn
}

THÔNG TIN

DOI: $10.46223 / \mathrm{HCMCOUJS}$. econ.vi.15.3.1334.2020

Ngày nhận: 20/04/2020

Ngày nhận lại: 04/06/2020

Duyệt đăng: 18/08/2020

Tù khóa:

giá trị chức năng, giá trị cảm xúc, giá trị tri thức, giá trị kinh tế, chấp nhận sản phẩm mới

Keywords:

functional value, hedonic value, epistemic value, economic value, new product adoption

\section{TÓM TÁ̃T}

Nghiên cứu này kiểm định tác động của các giá trị tiêu dùng đến ý định chấp nhận các sản phẩm điện tử cá nhân mới bao gồm thiết bi đeo, máy tính bảng và điện thoại thông minh. Nghiên cứu sử dụng bộ dữ liệu được thu thập từ 640 người tiêu dùng tại thành phố Hồ Chí Minh, mô hình cấu trúc tuyến tính (SEM) được áp dụng để kiểm định mô hình và các giả thuyết nghiên cứu. Kết quả nghiên cứu cho thấy các giá trị tiêu dùng có tác động đến ý định chấp nhận sản phẩm mới theo thứ tự giảm dần bao gồm: giá trị chức năng, giá trị cảm xúc, giá trị tri thức và giá trị kinh tế. Từ kết quả của đề tài, nhóm nghiên cứu đề xuất một số hàm ý quản trị trong việc gia tăng ý định chấp nhận sản phẩm mới của người tiêu dùng trong giai đoạn thương mại hóa sản phẩm.

\section{ABSTRACT}

This research examines the impact of consumption values on adoption of new personal electronics products such as wearable devices, tablets and smartphones. A data set was collected from 640 consumers in Ho Chi Minh City. Structural equation modeling (SEM) was adopted to test hypotheses. The results show four consumption values have positively related with the intention to adopt new personal electronic devices from the biggest impact to smallest one, including: functional value, hedonic value, epistemic value and economic value. From the results, this research proposed some implications for the sake of enhancing the understanding of consumers' new product adoption intention, especially during the product launch stage.

\section{Giới thiệu}

Sản phẩm mới có vai trò quan trọng với sự sống còn của doanh nghiệp tuy nhiên việc tung sản phẩm mới tiềm ẩm nhiều rủi ro và thách thức, đòi hỏi doanh nghiệp cần có hiểu biết sâu sắc về các yếu tố ảnh hưởng đến sự chấp nhận sản phẩm mới của người tiêu dùng. Theo $\mathrm{Im}$, Bhat, và Lee (2014), người tiêu dùng có xu hướng chấp nhận một sản phẩm mới dựa trên nhận thức của họ về giá trị mà sản phẩm mới mang lại. Việc sở hữu các sản phẩm nói chung hay sản phẩm mới nói 
riêng giúp cho người tiêu dùng đạt được các mục tiêu cá nhân hay nhóm mà họ đang theo đuổi (Caricati \& Raimondi, 2015; Grewal, Mehta, \& Kardes, 2000). Sheth, Newman, và Gross (1991) cũng cho rằng các giá trị tiêu dùng là yếu tố giải thích cho quyết định lựa chọn sản phẩm hay nhãn hiệu cụ thể. Hơn nữa, các giá trị tiêu dùng có thể khác biệt giữa các quốc gia và nền văn hóa (Sweeney \& Soutar, 2001), vì thế các doanh nghiệp không thể áp dụng chiến lược tung sản phẩm mới như nhau cho tất cả các thị trường (Lee, Kim, Lee, \& Kim, 2002). Wu và Chang (2016) cũng khẳng định nghiên cứu về tác động của giá trị tiêu dùng đến hành vi chấp nhận sản phẩm mới cần được thực hiện tại tại các quốc gia khác nhau vì giá trị tiêu dùng với một sản phẩm có thể được đánh giá khác nhau giữa những người tiêu dùng thuộc hai nền văn hóa, thậm chí có sự khác biệt giữa các nhánh trong cùng một nền văn hóa. Sản phẩm nào có giá trị tiêu dùng cao hơn thì cơ hội thị trường đối với sản phẩm đó sẽ lớn hơn (Tran, 2013). Chaudhuri, Aboulnasr, và Ligas (2010), Hong, Lin, và Hsieh (2016) cho rằng các nghiên cứu về giá trị tiêu dùng tuy được thực hiện nhiều nhưng còn quá ít các nghiên cứu về các giá trị tiêu dùng với nhóm sản phẩm mới vì thế cần nhiều nghiên cứu hơn để làm rõ vấn đề này. Về mặt thực tiễn, trên thị trường nhóm sản phẩm điện tử cá nhân được xem là nhóm sản phẩm chịu ảnh hưởng của công nghệ cao và được doanh nghiệp chú trọng phát triển sản phẩm mới hơn so với các sản phẩm khác (Chao, Reid, \& Mavondo, 2013). Các thiết bị cá nhân có thể được chia thành các nhóm như thiết bị di động bao gồm điện thoại thông minh, máy tính bảng, thiết bị đeo (wearable devices); nhóm thiết bị nghe nhìn như tivi, dàn âm thanh...etc. Theo báo cáo của công ty nghiên cứu thị trường GfK về thị trường sản phẩm công nghệ điện tử Việt Nam năm 2018, nhóm ngành hàng điện tử cá nhân là một trong bốn nhóm ngành hàng có sức tăng trưởng cao nhất với doanh thu 46,98 ngàn tỷ đồng; tăng 23,5\% so với năm 2017 , trong đó phải kể đến sự tăng trưởng của các thiết bị thông minh như điện thoại thông minh, thiết bị đeo thông minh và máy tính bảng. Thị trường các sản phẩm điện tử cá nhân được đánh giá rất sôi động khi hàng năm có rất nhiều sản phẩm mới được giới thiệu ra thị trường, tuy nhiên cũng nhiều sản phẩm mới không được thị trường chấp nhận. Chính vì vậy, nghiên cứu này kiểm định mối quan hệ giữa các giá trị tiêu dùng sản phẩm mới và hành vi chấp nhận các sản phẩm điện tử cá nhân mới tại thị trường thành phố Hồ Chí Minh là một đề tài cần thiết, có ý nghĩa khoa học và thực tiễn.

\section{Cơ sở lý thuyết}

\subsection{Các khái niệm nghiên cúu}

\subsubsection{Sản phẩm mới}

Quan điểm về sản phẩm mới (new product) rất đa dạng cũng như vẫn còn những tranh luận xung quanh câu hỏi thế nào là một sản phẩm mới. Nghiên cứu này tập trung tìm hiểu về các sản phẩm mới với doanh nghiệp bao gồm dòng sản phẩm mới hay sản phẩm được bổ sung, cải tiến dựa theo các phiên bản trước. Nhóm sản phẩm này chiếm trên $50 \%$ các sản phẩm mới trên thị trường (Booz-Allen \& Hamilton, 2005). Quan điểm này cùng phù hợp với tình hình thực tế tung các sản phẩm mới của trong nhóm sản phẩm điện tử cá nhân hiện nay. Các doanh nghiệp thường tung các sản phẩm cải tiến, nâng cấp từ các phiên bản trước, các sản phẩm này còn được gọi là các sản phẩn phiên bản mới (new version) hay thế hệ mới (new generation).

\subsubsection{Giá trị tiêu dùng}

Giá trị tiêu dùng là khái niệm quan trọng và được thảo luận cả trong thực tế và các nghiên cứu học thuật. Do có nhiều khái niệm khác nhau, các nhà nghiên cứu gặp khó khăn trong việc chọn khái niệm phù hợp nhất (Chang $\&$ Dibb, 2012). Theo quan điểm của Zeithaml (1988), giá trị tiêu dùng được hiểu là đánh giá tổng thể của người tiêu dùng về sản phẩm dựa trên sự so sánh về lợi ích hay tính hữu dụng của sản phẩm nhận được (get) với các chi phí hay tổn thất mà người đó phải 
đánh đổi (give). Theo Zeithaml (1988), giá trị tiêu dùng sẽ đạt được theo các cách thức sau: giá trị nghĩa là giá thấp, giá cả là tất cả những gì người tiêu dùng mong muốn từ sản phẩm, giá trị là chất lượng sản phẩm tương xứng với giá cả, và cuối cùng giá trị là tất cả những gì nhận được so với những thứ phải bỏ ra. Khái niệm của Zeithaml (1988) được nhiều nhà nghiên cứu đánh giá là đơn giản, dễ dàng đo lường thông qua việc hỏi đáp viên đánh giá về giá trị mà họ nhận được khi mua sản phẩm. Tuy nhiên, một số tác giả khác (Babin, Darden, \& Griffin, 1994; Holbrook, 1999; Sweeney \& Soutar, 2001) không đồng thuận với khái niệm này khi chỉ xem giá trị tiêu dùng đơn giản là sự so sánh giữa lợi ích và "sự hi sinh". Theo Tran (2013), giá trị tiêu dùng của một sản phẩm là đánh giá của khách hàng về khả năng cống hiến các lợi ích của nó nhằm thoản mãn nhu cầu và mong muốn của người tiêu dùng. Giá trị tiêu dùng có thể là các lợi ích, công dụng, giải pháp, cảm xúc do tiêu dùng sản phẩm mang lại chứ không phải là giá trị theo nghĩa chi phí (Tran, 2013). Như vậy, cách khái niệm và đo lường quá đơn giản sẽ gây khó khăn trong việc cung cấp các chỉ dẫn về cách tạo dựng giá trị tiêu dùng của sản phẩm (Chang \& Dibb, 2012; Zauner, Koller, \& Hatak, 2015). Khái niệm giá trị tiêu dùng nên được xem là khái niệm đa hướng, nghĩa là xem xét theo "các giá trị" (values) tiêu dùng chứ không đơn thuần là "giá trị" (value) (Sheth et al., 1991; Holbrook, 1999). Nhìn chung, các giá trị tiêu dùng tập trung vào các lợi ích về lý tính hay lý trí (cognitive) và cảm tính hay cảm xúc (affective) (Holbrook, 1999) hoặc thuộc về các giá trị chức năng (utilitarian) và giá trị tinh thần (hedonic) (Babin et al., 1994). Trong nghiên cứu này, giá trị tiêu dùng được định nghĩa là sự đánh giá của người tiêu dùng về khả năng của sản phẩm mới đáp ứng được các nhu cầu của họ (Tran, 2013), có vai trò định hướng và giải thích ý định chấp nhận sản phẩm mới của người tiêu dùng. Nghiên cứu chấp nhận khái niệm giá trị tiêu dùng theo quan điểm đa hướng, tương đồng với khái niệm của Sheth và cộng sự (1991) và được nhiều nghiên cứu chấp nhận và sử dụng trong các nghiên cứu về sản phẩm mới.

\subsection{3. Ý định chấp nhận sản phẩm}

Trong nghiên cứu hành vi người tiêu dùng nói chung, ý định thực hiện hành vi luôn là tiền đề của hành vi thực sự (Fishbein \& Ajzen, 2010). Thang đo hành vi ý định được nhiều nhà nghiên cứu kế thừa và chấp thuận vì hành vi thực sự là kết quả của ý định hành vi (Hassan, 2017). Ý định được cho là xu hướng của một người sẵn sàng dùng thử, có kế hoạch để phát triển và thực hiện các hành vi; ý định càng mạnh thì càng dễ dẫn đến hành vi thực sự (Ajzen, 2011). Ý định chấp nhận sản phẩm mới có thể được xem như mức độ cam kết và sẵn sàng dùng thử và tìm hiểu thêm về sản phẩm mới (Chaudhuri et al., 2010) hay thể hiện cam kết về ý định mua ngay sản phẩm khi nó xuất hiện trên thị trường (Hanzaee, Mina, \& Fatemeh, 2011). Dù có nhiều thang đo lường ý định chấp nhận sản phẩm mới nhưng thang đo ý định mua sản phẩm mới được nhiều nghiên cứu sử dụng nhất (Jeong, Kim, Park, \& Choi, 2016).

\subsection{Phát triển giả thuyết nghiên cưu}

Giá trị chức năng (functional value) là các lợi ích có được từ thuộc tính mà sản phẩm mang lại cũng như hiệu quả thực hiện các chức năng được kỳ vọng của sản phẩm (Sheth et al., 1991). Các nghiên cứu cũng cho thấy ngoài giá trị về chức năng (functional/utilitarian) thì người tiêu dùng cũng quan tâm đến giá trị tiêu khiển hay cảm xúc (hedonic) khi tiêu dùng sản phẩm mới (Vandecasteele \& Geuens, 2010). Giá trị tinh thần thường liên quan đến các giá trị về mặt cảm xúc mà cá nhân kỳ vọng. Giá trị cảm xúc (emotional value) cảm xúc thể hiện khả năng một sản phẩm hay dịch vụ tạo ra các cảm xúc hay trạng thái của người tiêu dùng. Thông thường, người tiêu dùng mong đợi những trải nghiệm hay cảm xúc nhất định nào đó thông qua tiêu dùng sản phẩm (Sheth et al., 1991). Kết quả nghiên cứu của Hur, Yoo, và Chung (2012) tại Hàn Quốc khẳng định giá trị chức năng, giá trị cảm xúc có ảnh hưởng đến ý định chấp nhận các sản phẩm công nghệ mới. Nghiên cứu của Caricati và Raimondi (2015) nhằm tìm hiểu mối quan hệ của các động cơ đổi mới 
và hành vi chấp nhận sản phẩm điện thoại thông minh (smartphone) mới tại Ý cho thấy kết quả nhóm động cơ về chức năng và cảm xúc đều ảnh hưởng đến ý định chấp nhận sản phẩm mới. Choi và Kim (2016) cũng phát hiện kết quả ảnh hưởng của nhận thức sự hữu ích và niềm vui đến ý định sử dụng thiết bị đeo là đồng hồ thông minh. Hong và cộng sự (2016) cũng cho rằng ý định chấp nhận sản phẩm mới là sản phẩm đồng hồ thông minh chịu ảnh hưởng bởi các giá trị nội tại và ngoại biểu của sản phẩm bao gồm giá trị chức năng và cảm xúc. Từ các cơ sở trên, nghiên cứu đề xuất giả thuyết sau:

$H_{1}$ : Giá trị chức năng có ảnh hưởng thuận chiều đến ý định chấp nhận sản phẩm điện tử cá nhân mới nhân mới

$H_{2}$ : Giá trị cảm xúc có ảnh hưởng thuận chiều đến ý định chấp nhận sản phẩm điện tử cá

Giá trị xã hội (social value) là giá trị có được thông qua mối liên hệ giữa sản phẩm với một hay một số tổ chức xã hội có liên quan đến người tiêu dùng. Giá trị xã hội hiện mối liên hệ tích cực hay tiêu cực với các nhóm liên quan đến văn hóa, tôn giáo, kinh tế xã hội (Sheth et al., 1991; Sweeney \& Soutar, 2001). Giá trị xã hội là yếu tố đáng chú ý hơn trong bối cảnh các quốc gia đang phát triển vì chủ nghĩa tập thể được xem trọng hơn tại các quốc gia này (Hofstede, 1980). Grewal và cộng sự (2000) xem giá trị xã hội mà sản phẩm mang lại như chức năng nhận dạng xã hội, có vai trò quan trọng trong việc giúp người tiêu dùng thể hiện và hòa nhập nhanh chóng với môi trường họ mong muốn. $\mathrm{O}^{\prime}$ Cass và Lim (2002) khẳng định rằng các sản phẩm mà cá nhân có thể mang theo cùng được xem như một biểu tượng của sự nhận dạng xã hội (social identity). Vì vậy, nghiên cứu đề xuất giả thuyết sau: nhân mới

H3: Giá trị xã hội có ảnh hưởng thuận chiều đến ý định chấp nhận sản phẩm điện tử cá

Giá trị tri thức (epistemic value) là khả năng một sản phẩm hay dịch vụ mang đến cho người tiêu dùng sự mới lạ hoặc sự thỏa mãn nhu cầu nâng cao hiểu biết của họ. Sheth và cộng sự (1991) cũng cho rằng rất cả các trải nghiệm hoàn toàn mới đều mang lại cho người dùng giá trị tri thức, tuy nhiên một sự thay đổi nhỏ trong sản phẩm, dịch vụ cũng có thể mang lại giá trị này. Giá trị tri thức được xem xét như một chức năng quan trọng ảnh hưởng đến hành vi, nhất là trong trường hợp sản phẩm và dịch vụ mới (Sweeney \& Soutar, 2001). Giá trị tri thức thôi thúc sự tò mò hay khát khao tìm kiếm và học hỏi những điều mới mẻ của những người tiêu dùng, vì thế giá trị tri thức có ảnh hưởng tích cực đến ý định chấp nhận sản phẩm mới (Bhatti, 2007; Pihlström \& Brush, 2008). Kết quả nghiên cứu của các tác giả khác như Hur và cộng sự (2012); Caricati và Raimondi (2015) cũng khẳng định giá trị tri thức có ảnh hưởng đến ý định chấp nhận các sản phẩm công nghệ mới. Vì vậy, nghiên cứu đề xuất giả thuyết sau: nhân mói

H4: Giá trị tri thức có ảnh hương thuận chiều đến ý định chấp nhận sản phẩm điện tử cá

Kotler và Amstrong (2012) cho rằng giá là nhân tố quan trọng trong phối thức marketing (marketing mix) và có ảnh hưởng đến quyết định mua của người tiêu dùng. Mô hình của Sheth và cộng sự (1991) xem giá trị về giá cả hay tiền tệ thuộc về giá trị chức năng nhưng Sweeney và Soutar (2001) cho rằng nhất thiết phải tách nhóm giá trị chức năng thành hai nhóm giá trị về chức năng và giá trị về giá cả của sản phẩm để đo lường riêng. Trên thực tế không thể phủ nhận vai trò của giá cả trong hành vi, nhất là trong quyết định mua một sản phẩm chứ không đơn thuần là sử dụng một dịch vụ miễn phí. Giá trị về giá cả (price) hay giá trị tính bằng tiền (money) còn được xem như giá trị kinh tế (economic) là các lợi ích nhận được từ sản phẩm giúp giảm thiểu chi phí trong ngắn hạn hay dài hạn (Sweeney \& Soutar, 2001). Hua, Le, và Le (2015) cho rằng lợi yếu tố 
giá cả là giá trị thực dụng mà người tiêu dùng mong muốn khi mua các sản phẩm điện máy. Vì thế, nghiên cứu cho rằng nếu các sản phẩm mới có giá cả phù hợp và sản phẩm mới đem lại giá trị kinh tế càng cao thì sẽ càng làm tăng ý định chấp nhận sản phẩm mới. Nghiên cứu đề xuất giả thuyết sau:

\section{H5: Giá trị kinh tế có ảnh hưởng tích cực đến ý định chấp nhận sản phẩm điện tử cá nhân mói}

\section{Phương pháp nghiên cứu}

\subsection{Mẫu}

Về qui mô mẫu, nghiên cứu sử dụng tiêu chuẩn để phân tích SEM nên cỡ mẫu tối thiểu cần khảo sát là 300 (Hair, Anderson, Babin, \& Black, 2010). Kết hợp với nguồn lực của nghiên cứu, nhóm thực hiện đề tài quyết định chọn cỡ mẫu tối thiểu là 500 , được chọn với phương pháp lấy mẫu thuận tiên. Sau khi khảo sát 700 người bằng kỹ thuật phỏng vấn trực tiếp, nhóm nghiên cứu thu được 640 bảng khảo sát hợp lệ để đưa vào nhập liệu và phân tích. Về đặc điểm mẫu khảo sát, tỷ lệ giới tính tham gia vào mẫu tương đối đồng đều với $52,5 \%$ nam và $47,5 \%$ là nữ. Về độ tuổi, nhóm từ 18 đến 25 tuổi chiếm 36,6\%; từ 26 đến 35 chiếm 35,9\%; từ 36 đến 45 chiếm 19,2\% và trên 45 chiếm $8,3 \%$.

\subsection{Thang đo các khái niệm nghiên cứu}

Các thang đo trong mô hình được kế thừa từ các nghiên cứu trước đây, được điều chỉnh cho phù hợp về mặt ngữ nghĩa thông qua thảo luận nhóm với 12 người tiêu dùng có tuổi từ 18 trở lên tại thành phố Hồ Chí Minh. Kết quả của thảo luận nhóm có được thang đo ban đầu để tiến hành nghiên cứu sơ bộ. Trước khi khảo sát, bảng câu hỏi được gửi đến hai chuyên gia là giảng viên ngành Marketing để đánh giá về nội dung và hình thức bảng câu hỏi. Nghiên cứu định lượng sơ bộ với 120 quan sát nhằm mục đích chính là đánh giá thang đo thông qua tính hệ số Cronbach's Alpha, phân tích nhân tố khám phá (EFA). Kết quả định lượng sơ bộ cho thấy 5 nhân tố độc lập và 1 nhân tố phụ thuộc được giữ lại theo tiêu chí Cronbach's Alpha $(\alpha)$ của mỗi nhân tố trên 0,7 và các biến quan sát có hệ số tải nhân tố $(\mathrm{FL})$ trên 0,5 (Hair et al., 2010). Các thang đo được giữ lại sẽ sử dụng để đưa vào bảng câu hỏi chính thức, các mục hỏi (phát biểu) được hỏi với thang Likert với năm mức độ tương ứng (1 - Rất không đồng ý; 5 - Rất đồng ý). Các thang đo này sẽ tiếp tục đánh giá về độ tin cậy và giá trị của thang đo trong nghiên cứu định lượng chính thức với cỡ mấu là 640 .

\section{Bảng 1}

Bảng tổng hợp thang đo sau nghiên cứu sơ bộ

\begin{tabular}{|c|c|c|c|}
\hline Thang đo & Ký hiệu & Diễn giải biến quan sát & Nguồn \\
\hline \multirow{3}{*}{$\begin{array}{l}\text { Giá trị } \\
\text { chức } \\
\text { năng }\end{array}$} & FUNC1 & $\begin{array}{l}\text { Sản phẩm mới hữu ích cho công việc và cuộc sống } \\
\text { của tôi }\end{array}$ & \multirow{3}{*}{$\begin{array}{l}\text { Sweeney và Soutar } \\
(2001) \text {; Hur và cộng } \\
\text { sự }(2012)\end{array}$} \\
\hline & FUNC2 & $\begin{array}{l}\text { Sản phẩm mới có nhiều ưu việt hơn so với sản phẩm } \\
\text { hiện tại }\end{array}$ & \\
\hline & FUNC3 & Sản phẩm mới thường có thêm nhiều tính năng mới & \\
\hline \multirow{2}{*}{$\begin{array}{l}\text { Giá trị } \\
\text { cảm xúc }\end{array}$} & HEDO1 & $\begin{array}{l}\text { Sử dụng sản phẩm mới đem lại cho tôi niềm vui cá } \\
\text { nhân }\end{array}$ & \multirow{2}{*}{$\begin{array}{l}\text { Sweeney và Soutar } \\
\text { (2001); Hur và cộng } \\
\text { sự (2012) }\end{array}$} \\
\hline & HEDO2 & $\begin{array}{l}\text { Sản phẩm mới khiến cuộc sống của tôi thú vị và sôi } \\
\text { động hơn }\end{array}$ & \\
\hline
\end{tabular}




\begin{tabular}{|c|c|c|c|}
\hline Thang đo & Ký hiệu & Diễn giải biến quan sát & Nguồn \\
\hline & HEDO3 & $\begin{array}{l}\text { Sản phẩm mới giúp tôi thỏa mãn hơn trong cuộc } \\
\text { sông }\end{array}$ & \\
\hline \multirow{4}{*}{$\begin{array}{l}\text { Giá trị } \\
\text { xã hội }\end{array}$} & SOC1 & $\begin{array}{l}\text { Sở hữu sản phẩm mới giúp tội được mọi người thừa } \\
\text { nhận }\end{array}$ & \multirow{4}{*}{$\begin{array}{l}\text { Sheth và cộng sự } \\
\text { (1991); Sweeney và } \\
\text { Soutar } \\
\text { (2001); Hur và cộng } \\
\text { sự (2012) }\end{array}$} \\
\hline & SOC2 & $\begin{array}{l}\text { Sở hữu sản phẩm mới giúp tôi cải thiện mối quan hệ } \\
\text { với mọi người }\end{array}$ & \\
\hline & SOC3 & Sản phẩm mới giúp tôi gây ấn tượng với người khác & \\
\hline & SOC4 & Sử dụng sản phẩm mới giúp tôi tự tin hơn & \\
\hline \multirow{4}{*}{$\begin{array}{l}\text { Giá trị } \\
\text { tri thức }\end{array}$} & EPIS1 & Sản phẩm mới cho tôi nhiều trải nghiệm mới mẻ & \multirow{4}{*}{$\begin{array}{l}\text { Sheth và cộng sự } \\
\text { (1991); Hur và cộng } \\
\text { sự (2012) }\end{array}$} \\
\hline & EPIS2 & Sản phẩm mới giúp tôi tư duy tốt hơn & \\
\hline & EPIS3 & Sản phẩm mới kích thích trí tò mò của tôi & \\
\hline & EPIS4 & $\begin{array}{l}\text { Sản phẩm mới giúp tôi cập nhật kiến thức mới về } \\
\text { công nghệ }\end{array}$ & \\
\hline \multirow{3}{*}{$\begin{array}{l}\text { Giá trị } \\
\text { kinh tế }\end{array}$} & ECO1 & Sản phẩm điện tử cá nhân mới có giá cả phù hợp & \multirow{3}{*}{$\begin{array}{l}\text { Sweeney và Soutar } \\
(2001)\end{array}$} \\
\hline & $\mathrm{ECO} 2$ & $\begin{array}{l}\text { Giá sản phẩm mới thường tương xứng với chất } \\
\text { lượng }\end{array}$ & \\
\hline & $\mathrm{ECO} 3$ & $\begin{array}{l}\text { Sử dụng sản phẩm hỗ trợ tôi đạt được các lợi ích kinh } \\
\text { tế }\end{array}$ & \\
\hline \multirow{4}{*}{$\begin{array}{l}\text { Ý định } \\
\text { chấp } \\
\text { nhận }\end{array}$} & INT1 & Tôi nghĩ mua sản phẩm mới là lựa chọn đúng đắn & \multirow{4}{*}{$\begin{array}{l}\text { Alexander, Lynch, } \\
\text { và Wang (2008); } \\
\text { Jeong và cộng sự } \\
(2016)\end{array}$} \\
\hline & INT2 & $\begin{array}{l}\text { Tôi chắc chắn sẽ mua ngay sản phẩm mới khi nó có } \\
\text { mặt trên thị trường }\end{array}$ & \\
\hline & INT3 & $\begin{array}{l}\text { Nếu tôi cần mua một thiết bị điện tử cá nhân, tôi sẽ } \\
\text { mua mẫu mới nhất }\end{array}$ & \\
\hline & INT4 & $\begin{array}{l}\text { Tôi sẽ nỗ lực hết mình để có thể sở hữu và sử dụng } \\
\text { sản phẩm điện tử cá nhân mới }\end{array}$ & \\
\hline
\end{tabular}

Nguồn: Tổng hợp và điều chỉnh từ nghiên cứu sơ bộ của nhóm nghiên cứu

\section{Kết quả và thảo luận}

\subsection{Kết quả phân tích thang đo}

\subsubsection{Kết quả hệ số Cronbach’ Alpha và EFA}

Kết quả kiểm định thang đo bằng hệ số Cronbach's Alpha sử dụng tiêu chuẩn về hệ số Cronbach's Alpha của các nhân tố lớn hơn 0,7 thể hiện thang đo có mức độ đo lường tốt, hệ số tương quan biến-tổng đều lớn hơn 0,3 . Toàn bộ các thang đo tiếp tục được đưa vào phân tích EFA. Kết quả phân tích nhân tố khám phá với phép rút PAF và phép xoay Promax cho thấy các điều kiện để thực hiện EFA là phù hợp, trong đó hệ số $\mathrm{KMO}=0,811$ (lớn hơn 0,5 ); số Sig của kiểm định Bartlett's $=0,00$ (nhỏ hơn 0,05 ). Sáu nhân tố rút được tại điểm dừng Eigenvalue $=1,143$ (lớn hơn 1), tổng phương sai trích đạt 62,348\% (lớn hơn 50\%). Kết quả EFA cho thấy một biến quan sát thuộc thang đo giá trị tri thức, thể hiện qua phát biểu "Sản phẩm mới cho tôi nhiều trải nghiệm mới mẻ” (EPIS1) bị loại vì có hệ số tải nhân tố thấp hơn 0,5 . Các biến quan sát khác đạt yêu cầu 
về hệ số tải nhân tố, dao động từ 0,639 đến 0,932 được đưa vào các phân tích tiếp theo.

\subsubsection{Kết quả kiểm định thang đo bằng $C F A$}

Các khái niệm nghiên cứu sau EFA đạt yêu cầu được đưa vào phân tích nhân tố khẳng định (CFA) nhằm khẳng định lại độ tin cậy và giá trị của mô hình đo lường. Tiêu chí đánh giá bao gồm tính đơn hướng, độ tin cậy của thang đo, giá trị hội tụ và giá trị phân biệt. Kết quả của ước lượng bằng phương pháp ước lượng hợp lý cực đại (ML) cho kết quả như sau: Chi-square/df = 3,387 có thể chấp nhận được vì nhỏ hơn 5 (Bollen, 1989); các giá trị $\mathrm{CFI}=0,931$; GFI $=0,913$ và $\mathrm{TLI}=$ 0,915 đều cao hơn 0,9 ; RMSEA $=0,061$ (nhỏ hơn 0,08 ) nên mô hình phù hợp với tập dữ liệu (Hair et al., 2010). Sự phù hợp của mô hình cũng là điều kiện cần và đủ để xác định một tập các mục hỏi có đạt tính đơn hướng (Steenkamp \& van Trijp, 1991). Kết quả bảng 2 cho thấy hệ số độ tin cậy tổng hợp $(\mathrm{CR})$ có giá trị từ 0,788 đến 0,871 (lớn hơn 0,5 ) cho thấy thang đo đạt độ tin cậy. Tiếp theo, giá trị hội tụ của thang đo được đánh giá thông qua hệ số tải chuẩn hóa của các biến quan sát và phương sai trích trung bình (AVE). Hệ số tải chuẩn hóa của các biến quan sát từ 0,652 đến 0,936 đều đạt trên 0,5 (Hair et al., 2010) và phương sai trích trung bình (AVE) được sử dụng để đánh giá lại độ hội tụ bên trong của các mục hỏi trong cùng một khái niệm trong khoảng 0,555 đến 0,692 (lớn hơn 0,5 ) vì thế các thang đo thoả mãn giá trị hội tụ (Bagozzi \& Yi, 1988). Kết quả kiểm định giá trị phân biệt của thang đo cho thấy các hệ số tương quan giữa các khái niệm nghiên cứu nhỏ hơn 1 , có ý nghĩa thống kê $(\mathrm{p}=0.00<0.05)$. Bên cạnh đó tiêu chuẩn Fornell và Lacker (1981) cho thấy AVE của một khái niệm đều lớn hơn tương quan bình phương cao nhất (MSV) của nó với khái niệm khác, như vậy các thang đo đều đạt giá trị phân biệt.

\section{Bảng 2}

Kết quả đánh giá mô hình đo lường

\begin{tabular}{|c|c|c|c|c|c|c|c|}
\hline \multirow{2}{*}{ Nhân tố } & \multirow{2}{*}{ Biến quan sát } & \multicolumn{2}{|c|}{ Hệ số tải nhân tố } & \multirow{2}{*}{ Hệ số $\alpha$} & \multirow{2}{*}{$\begin{array}{l}\text { Độ tin cây tổng } \\
\text { hợp (CR) }\end{array}$} & \multirow{2}{*}{ AVE } & \multirow{2}{*}{ MSV } \\
\hline & & EFA & CFA & & & & \\
\hline \multirow{3}{*}{$\begin{array}{l}\text { Giá trị } \\
\text { chức } \\
\text { năng }\end{array}$} & FUNC1 & 0,745 & 0,815 & \multirow{3}{*}{0,800} & \multirow{3}{*}{0,807} & \multirow{3}{*}{0,586} & \multirow{3}{*}{0,415} \\
\hline & FUNC2 & 0,821 & 0,829 & & & & \\
\hline & FUNC3 & 0,662 & 0,638 & & & & \\
\hline \multirow{3}{*}{$\begin{array}{l}\text { Giá trị } \\
\text { cảm xúc }\end{array}$} & HEDO1 & 0,925 & 0,889 & \multirow{3}{*}{0,868} & \multirow{3}{*}{0,871} & \multirow{3}{*}{0,692} & \multirow{3}{*}{0,140} \\
\hline & HEDO2 & 0,741 & 0,794 & & & & \\
\hline & HEDO3 & 0,817 & 0,810 & & & & \\
\hline \multirow{4}{*}{$\begin{array}{l}\text { Giá trị } \\
\text { xã hội }\end{array}$} & SOC1 & 0,645 & 0,652 & \multirow{4}{*}{0,852} & \multirow{4}{*}{0,858} & \multirow{4}{*}{0,609} & \multirow{4}{*}{0,013} \\
\hline & SOC2 & 0,734 & 0,730 & & & & \\
\hline & SOC3 & 0,784 & 0,777 & & & & \\
\hline & SOC4 & 0,932 & 0,936 & & & & \\
\hline \multirow{3}{*}{$\begin{array}{l}\text { Giá trị } \\
\text { tri thức }\end{array}$} & EPIS2 & 0,789 & 0,799 & \multirow{3}{*}{0,851} & \multirow{3}{*}{0,852} & \multirow{3}{*}{0,657} & \multirow{3}{*}{0,189} \\
\hline & EPIS3 & 0,839 & 0,817 & & & & \\
\hline & EPIS4 & 0,774 & 0,817 & & & & \\
\hline \multirow{3}{*}{$\begin{array}{l}\text { Giá trị } \\
\text { kinh tế }\end{array}$} & ECO1 & 0,804 & 0,825 & \multirow{3}{*}{0,782} & \multirow{3}{*}{0,788} & \multirow{3}{*}{0,555} & \multirow{3}{*}{0,189} \\
\hline & $\mathrm{ECO} 2$ & 0,648 & 0,656 & & & & \\
\hline & ECO3 & 0,765 & 0,745 & & & & \\
\hline \multirow{4}{*}{$\begin{array}{l}\text { Ý định } \\
\text { chấp } \\
\text { nhận }\end{array}$} & INT1 & 0,833 & 0,813 & \multirow{4}{*}{0,858} & & & \\
\hline & INT2 & 0,837 & 0,796 & & 0844 & 0577 & 0415 \\
\hline & INT3 & 0,639 & 0,725 & & 0,047 & ון ונסינ & 0,411 \\
\hline & INT4 & 0,748 & 0,697 & & & & \\
\hline
\end{tabular}

Nguồn: Tính toán của nhóm tác giả 


\subsection{Kết quả phân tích mô hình cấu trúc}

Mô hình cấu trúc tuyến tính có các chỉ tiêu Chi-square/df $=3,805<5 ; \mathrm{CFI}=0,932 ; \mathrm{GFI}=$ 0,916; TLI = 0,915; RMSEA = 0,066 đạt yêu cầu chứng tỏ mô hình lý thuyết phù hợp với dữ liệu thực tế. Kết quả phân tích mô hình cấu trúc được thể hiện trong bảng 3 . Từ kết quả SEM ước lượng mô hình chuẩn hóa cho thấy giả thuyết $\mathrm{H}_{3}$ bị bác bỏ vì không có ý nghĩa thống kê; các giả thuyết $\mathrm{H}_{1}, \mathrm{H}_{2}, \mathrm{H}_{4}, \mathrm{H}_{5}$ được chấp nhận tại mức ý nghĩa 0,01 . Các hệ số ước lượng mối quan hệ được phát biểu trong các giả thuyết $\mathrm{H}_{1}, \mathrm{H}_{2}, \mathrm{H}_{4}, \mathrm{H}_{5}$ đều mang dấu dương, do đó các giả thuyết được chấp nhận đúng với quy ước ban đầu và đều có ý nghĩa thống kê. Theo đó, các biến độc lập bao gồm giá trị chức năng, giá trị cảm xúc, giá trị tri thức và giá trị kinh tế đều có ảnh hưởng tích cực (thuận chiều) đến ý định chấp nhận sản phẩm mới. Kết quả các hệ số hồi quy chuẩn hóa khẳng định giá trị chức năng (FUNC) là nhân tố tác động mạnh nhất đến ý định chấp nhận sản phẩm mới, thứ tự tiếp theo được sắp xếp theo mức độ tác động giảm dần là giá trị cảm xúc (HEDO); giá trị tri thức (EPIS) và giá trị kinh tế (ECO). Hệ số SMC (Squared Multiple Correlations) là 0,534 cho thấy các nhân tố độc lập trong mô hình giải thích được $53,4 \%$ biến thiên của biến phụ thuộc là ý định chấp nhận sản phẩm mới.

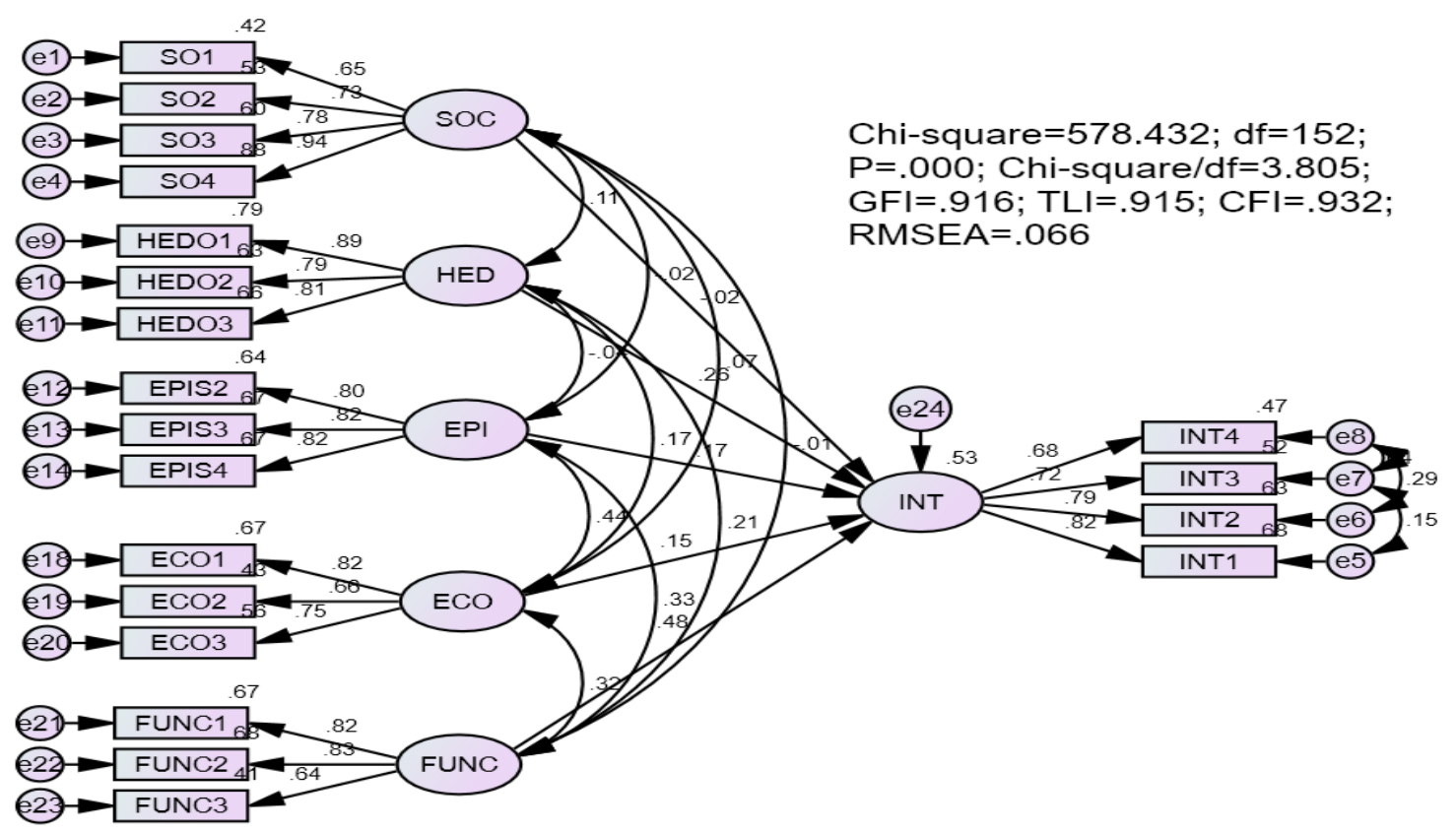

Hình 1. Kết quả phân tích SEM

Nguồn: Kết quả phân tích từ phần mềm AMOS

\section{Bảng 3}

Kết quả kiểm định các giả thuyết nghiên cứu

\begin{tabular}{|c|c|c|c|c|c|c|c|}
\hline \multicolumn{3}{|c|}{ Mối quan hệ } & \multirow{2}{*}{$\frac{\text { Hệ số }}{0,484}$} & \multirow{2}{*}{$\begin{array}{c}\begin{array}{c}\text { Sai } \\
\text { lệch }\end{array} \\
0,050\end{array}$} & \multirow{2}{*}{$\begin{array}{c}\begin{array}{c}\text { Giá trị } \\
\text { tới hạn }\end{array} \\
10,215\end{array}$} & \multirow{2}{*}{$\begin{array}{c}\text { P-value } \\
0,000\end{array}$} & \multirow{2}{*}{$\begin{array}{c}\text { Kết luận } \\
\text { Chấp nhận } H_{1}\end{array}$} \\
\hline $\begin{array}{l}\text { Giá trị chức } \\
\text { năng }\end{array}$ & $\rightarrow$ & $\begin{array}{l}\text { Ý định chấp nhận } \\
\text { sản phẩm (SP) mới }\end{array}$ & & & & & \\
\hline $\begin{array}{l}\text { Giá trị cảm } \\
\text { xúc }\end{array}$ & $\rightarrow$ & $\begin{array}{l}\text { Ý định chấp nhận } \\
\text { SP mới }\end{array}$ & 7 & 0,033 & 6,443 & 00 & Chấp nhận $\mathrm{H}_{2}$ \\
\hline Giá trị xã hội & $\rightarrow$ & $\begin{array}{l}\text { Ý định chấp nhận } \\
\text { SP mới }\end{array}$ & $-0,015$ & 0,087 & $-0,422$ & 0,673 & Bác bỏ $\mathbf{H}_{3}$ \\
\hline Giá trị tri thức & $\rightarrow$ & Ý định chấp nhận & 0,168 & 0,047 & 3,691 & 0,000 & Chấp nhận $\mathrm{H}_{4}$ \\
\hline
\end{tabular}




\begin{tabular}{|c|c|c|c|c|c|c|c|}
\hline \multicolumn{3}{|c|}{ Mối quan hệ } & \multirow[t]{2}{*}{ Hệ số } & \multirow[t]{2}{*}{$\begin{array}{l}\text { Sai } \\
\text { lệch }\end{array}$} & \multirow[t]{2}{*}{$\begin{array}{l}\text { Giá trị } \\
\text { tới hạn }\end{array}$} & \multirow[t]{2}{*}{ P-value } & \multirow[t]{2}{*}{ Kết luận } \\
\hline & & SP mới & & & & & \\
\hline Giá trị kinh tế & $\rightarrow$ & $\begin{array}{l}\text { Ý định chấp nhận } \\
\text { SP mới }\end{array}$ & 0,148 & 0,057 & 3,174 & 0,002 & Chấp nhận $\mathrm{H}_{5}$ \\
\hline
\end{tabular}

Nguồn: Kết quả phân tích từ phần mềm AMOS

\section{Thảo luận và khuyến nghị}

Đề tài tập trung vào việc đánh giá tác động của các giá trị tiêu dùng đến ý định chấp nhận sản phẩm mới với nhóm sản phẩm điện tử cá nhân tại thành phố Hồ Chí Minh, Việt Nam. Kết quả cho thấy có mối quan hệ tích cực giữa các giá trị tiêu dùng đến ý định chấp nhận sản phẩm mới theo thứ tự giảm dần bao gồm: giá trị chức năng, giá trị cảm xúc, giá trị kinh tế và giá trị tri thức. Kết quả nghiên cứu này ủng hộ các kết quả nghiên cứu của Caricati và Raimondi (2015); Vandecasteele và Geuens (2010) liên quan đến các động cơ thúc đẩy hành vi ý định mua sản phẩm mới. Giá trị xã hội không ảnh hưởng đến ý định chấp nhận sản phẩm mới, kết quả này tương đồng của với kết quả của Hur và cộng sự (2012). Như vậy, có thể thấy rằng, người tiêu dùng tại thành phố Hồ Chí minh có xu hướng độc lập hơn trong các quyết định, dám thể hiện mình và dám đi ngược với số đông. Kết quả này không đồng thuận với quan điểm của Hofstede (1980) cho rằng chủ nghĩa tập thể hay hành vi theo đám đông là đặc điểm văn hóa của người tiêu dùng tại các thị trường mới nổi. Nhìn chung, kết quả nghiên cứu phù hợp và ủng hộ quan điểm và kết quả nghiên cứu về yếu tố giá trị tinh thần (hedonic) và yếu tố lợi ích (utilitarian) ảnh hưởng đến hành vi tiêu dùng một sản phẩm (Holbrook, 1999).

Từ các kết quả trên, nhóm nghiên cứu đề xuất một số gợi ý giải pháp cho các doanh nghiệp sản xuất và kinh doanh các sản phẩm điện tử cá nhân nhằm gia tăng ý định chấp nhận sản phẩm mới trong giai đoạn thương mại hóa sản phẩm. Thứ nhất, các doanh nghiệp nên tập trung vào giá trị chức năng của sản phẩm điện tử cá nhân, người tiêu dùng luôn mong muốn các tính năng mới và vượt trội hơn so với các sản phẩm hiện tại. Để có thể đáp ứng nhu cầu này của người tiêu dùng, các doanh nghiệp cần không ngừng cập nhật công nghệ mới và ghi nhận các vấn đề về sản phẩm hiện tại để có thể cải tiến và nâng cấp các sản phẩm hiện có. Ngoài cung cấp giá trị về chức năng, các sản phẩm mới cũng cần gia tăng các trải nghiệm về mặt cảm xúc cho khách hàng khi tương tác với sản phẩm trong quá trình mua và sử dụng. Giá trị tri thức là những mong muốn trải nghiệm và học hỏi những điều mới, các doanh nghiệp hãy truyền thông đến khách hàng của mình về các lợi ích này, kích thích trí tò mò và thử thách về mặt trí tuệ của người sử dụng. Với một sản phẩm mới, chiến lược định giá cũng cần được nghiên cứu thật kỹ và có thể liên kết với các nhà phân phối hay công ty dịch vụ tài chính để vấn đề về giá cả không phải là rào cản của người tiêu dùng. Ngoài ra, chiến lược định giá theo dòng sản phẩm có thể được áp dụng, các doanh nghiệp nên có nhiều phân khúc khác nhau đáp ứng nhu cầu đa dạng của khách hàng.

Hạn chế của nghiên cứu này là chỉ tập trung đánh giá tác động của các giá trị tiêu dùng đến ý định chấp nhận sản phẩm mới mà chưa tìm hiểu các yếu tố tác động khác. Nghiên cứu cũng chưa xem xét mối quan hệ giữa các nhóm giá trị tiêu dùng, vì thực tế các giá trị này có thể có mối quan hệ với nhau (Sweeney \& Soutar, 2001). Các nghiên cứu tiếp theo có thể xem xét thêm các yếu tố khác tác động đến ý định chấp nhận sản phẩm mới như các yếu tố thuộc về tính cách cá nhân hay sự trung thành của khách hàng. Việc chọn mẫu theo thuận tiện cũng xem là một hạn chế về tính đại diện của kết quả. Hơn nữa, nghiên cứu chỉ thực hiện khảo sát tại khu vực nội thành thành phố Hồ Chí Minh, nơi người tiêu dùng được đánh giá là cởi mở và năng động hơn các vùng miền khác. Các nghiên cứu tiếp theo có thể thực hiện ở các địa phương khác để mở rộng phạm vi áp dụng của mô hình nghiên cứu cũng như so sánh sự khác biệt giữa các vùng miền tại Việt Nam. 


\section{Tài liệu tham khảo}

Ajzen, I. (2011). The theory of planned behaviour: Reactions and reflections. Psychology \& Health, 26(9), 1113-1127. doi:10.1080/08870446.2011.613995.

Alexander, D. L., Lynch, J. G., Jr., \& Wang, Q. (2008). As time goes by: Do cold feet follow warm intentions for really new versus incrementally new products. Journal of Marketing Research, 45(3), 307-319. doi:10.1509/jmkr.45.3.307

Babin, B. J., Darden, W. R., \& Griffin, M. (1994). Work and/or fun: Measuring hedonic and utilitarian shopping value. Journal of Consumer Research, 20(4), 644-654.

Bagozzi, R. P., \& Yi, Y. (1988). On the evaluation of structural equation models. Journal of The Academy of Marketing Science, 16(1), 74-94.

Bhatti, T. (2007). Exploring factors influencing the adoption of mobile commerce. Journal of Internet Banking and Commerce, 12(3), 1-13.

Booz-Allen \& Hamilton, A. (2005). New product management for the Lagos. New York, NY: Addison Wesley.

Caricati, L., \& Raimondi, M. (2015). The motivated consumer innovativeness scale: Initial Italian validation. Testing, Psychometrics, Methodology in Applied Psychology, 22(3), 363-383.

Chang, C., \& Dibb, S. (2012). Reviewing and conceptualising customer-perceived value. Marketing Review, 12(3), 253-274.

Chao, C. W., Reid, M., \& Mavondo, F. (2013). Global consumer innovativeness and consumer electronic product adoption. Asia Pacific Journal of Marketing and Logistics, 25(4), 614-630.

Chaudhuri, A., Aboulnasr, K., \& Ligas, M. (2010). Emotional responses on initial exposure to a hedonic or utilitarian description of a radical innovation. Journal of Marketing Theory and Practice, 18(4), 339-359.

Choi, J. R., \& Kim, S. (2016). Is the smartwatch an IT product or a fashion product? A study on factors affecting the intention to use smartwatches. Computers in Human Behavior, 63, 777786. doi:10.1016/j.chb.2016.06.007

Fishbein, M., \& Ajzen, I. (2010). Predicting and changing behavior: The reasoned action approach. New York, NY: Psychology Press.

Fornell, C. D., \& Lacker, D. F. (1981). Evaluating structural equation models with unobservable variables and measurement error. Journal of Marketing Research, 18(1), 39-50.

GfK. (2018). Global smartphone average sales price sees record year-on-year growth in 4Q17. Retrieved March 20, 2020, from https://www.gfk.com/press/global-smartphone-averagesales-price-sees-record-year-on-year-growth-in-4q17

Goldsmith, R. E., Clark, R. A., \& Goldsmith, E. B. (2007). The desire for unique consumer products, innovativeness, and conformity. Proceedings of the 2007 Academy of Marketing Science (AMS) Annual Conference, Coral Cables, Florida, 206-210.

Grewal, R., Mehta, R., \& Kardes, F. R. (2000). The role of the social-identity function of attitudes in consumer innovativeness and opinion leadership. Journal of Economic Psychology, 21(3), 233-252. 
Hair, J. F., Anderson, R. E., Babin, B. J., \& Black, W. C. (2010). Multivariate data analysis: A global perspective. Hoboken, NJ: Pearson.

Hanzaee, K. H., Mina, M. A., \& Fatemeh, A. (2011). Investigating the effect of gender role attitude on the relationship between dimensions of religiosity and new product adoption intention. World Applied Sciences Journal, 13(6), 1527-1536.

Hassan, H. S. (2017). The role of customer innovativeness in the new products adoption intentions: An empirical study on mobile phone customers of the Egyptian universities students. International Business Research, 10(4), 117-130.

Hofstede, G. (1980). Culture's consequences: International differences in work-related values. Beverly Hills, CA: Sage.

Holbrook, M. (1999). Consumer value: A framework for analysis and research. London, UK: Routledge.

Hong, J., Lin, P., \& Hsieh, P. (2016). The effect of consumer innovativeness on perceived value and continuance intention to use smartwatch. Computers in Human Behavior, 67, 264-272.

Hua, M. K. P., Le, L. P., \& Le, H. N. (2015). Định hướng giá trị khi đi mua sắm của người tiêu dùng đối với các kênh phân phối hiện đại: Một nghiên cứu tại các siêu thị điện máy Thành phố Hồ Chí Minh [The value orientation of consumer shopping for modern distribution channels: A study at electronics supermarkets in Ho Chi Minh City]. Tạp chí khoa hoc truò̀ng Đại học Mở Thành phố Hồ Chí Minh, 3(42), 111-120.

Hur, W. M., Yoo, J. J., \& Chung, T. L. (2012). The consumption values and consumer innovativeness on convergence products. Industrial Management \& Data Systems, 112(5), 688-706.

Im, S., Bhat, S., \& Lee, Y. (2014). Consumer perceptions of product creativity, coolness, value and attitude. Journal of Business Research, 68(1), 166-172. doi:10.1016/j.jbusres.2014.03.014

Jeong, S. C., Kim, S., Park, J. Y., \& Choi, B. (2016). Domain-specific innovativeness and new product adoption: A case of wearable devices. Telematics and Informatics, 34(5), 399-412.

Kotler, P., \& Armstrong, G. (2012). Principles of marketing (14th ed.). Upper Saddle River, NJ: Prentice Hall.

Lee, Y., Kim, J., Lee, I., \& Kim, H. (2002). A cross-cultural study on the value structure of mobile Internet usage: Comparison between Korea and Japan. Journal of Electronic Commerce Research, 3, 227-239.

Lu, J., Yao, J., \& Yu, C. (2005). Personal innovativeness, social influences and adoption of wireless Internet services via mobile technology. Journal of Strategic Information Systems, 14(3), 245-268.

O'Cass, A., \& Lim, K. (2002). Toward understanding the young consumer's brand associations and ethnocentrism in the Lion's port. Psychology and Marketing, 19(9), 759-775.

Pihlström, M., \& Brush, G. J. (2008). Comparing the perceived value of information and entertainment mobile services. Psychology \& Marketing, 25(8), 732-755.

Sheth, J. N., Newman, B. I., \& Gross, L. G. (1991). Why we buy what we buy: A theory of consumption values. Journal of Business Research, 22(2), 159-170. 
Steenkamp, J. E. B. M., \& van Trijp, J. C. M. (1991). The use of lisrel in validating marketing constructs. International Journal of Research in Marketing, 8(4), 283-299.

Sweeney, J. C., \& Soutar, G. N. (2001). Consumer perceived value: the development of multiple item scale. Journal of Retailing, 77(2), 203-220.

Tran, D. M. (2013). Giáo trình Marketing căn bản [Basic Marketing Curriculum]. Hanoi, Vietnam: NXB Đại học Kinh tế Quốc dân.

Vandecasteele, B., \& Geuens, M. (2010). Motivated consumer innovativeness: Concept, measurement, and validation. International Journal of Research in Marketing, 27(4), 308-318.

Wu, S., \& Chang, H. (2016). The model of relationship between the perceived values and the purchase behaviors toward innovative products. Journal of Management and Strategy, 7(2), 31-45.

Zauner, A., Koller, M., \& Hatak, I. (2015). Customer perceived value: Conceptualization and avenues for future research. Cogent Psychology, 2(1), 1-17.

Zeithaml, V. A. (1988). Consumer perceptions of price, quality and value: A mean-end model and synthesis of evidence. Journal of Marketing, 52(3), 2-22. 